\begin{tabular}{l|l} 
REVISTA & $\begin{array}{l}\text { Revista Educación } \\
\text { ISSN: 0379-7082 } \\
\text { ISSN: 2215-2644 } \\
\text { revedu@ gmail.com } \\
\text { Universidad de Costa Rica } \\
\text { Costa Rica }\end{array}$
\end{tabular}

\title{
La educación secundaria mexicana: Entre la búsqueda del acceso equitativo y el rezago $^{[1]}$
}

Ducoing Watty, Patricia

La educación secundaria mexicana: Entre la búsqueda del acceso equitativo y el rezago [1]

Revista Educación, vol. 42, núm. 2, 2018

Universidad de Costa Rica, Costa Rica

Disponible en: http://www.redalyc.org/articulo.oa?id=44055139031

DOI: https://doi.org/10.15517/revedu.v42i2.27665

Esta obra está bajo una Licencia Creative Commons Atribución-NoComercial-SinDerivar 3.0 Internacional. 


\title{
La educación secundaria mexicana: Entre la búsqueda del acceso equitativo y el
}

\author{
rezago $^{[1]}$ \\ Patricia Ducoing Watty [2] \\ Universidad Nacional Autónoma de México, México \\ pducoingw@gmail.com
}

Mexican Secondary Education: Between the Search for Equitable Access and Educational Lag

DOI: https://doi.org/10.15517/revedu.v42i2.27665

Redalyc: http://www.redalyc.org/articulo.oa?id=44055139031

Recepción: 10 Enero 2017

Aprobación: 16 Mayo 2018

\section{RESUMEN:}

Este trabajo representa un acercamiento a la situación de la educación secundaria mexicana, la cual ha venido experimentando, en las últimas décadas, una tensión entre la ampliación de la cobertura para todos los grupos adolescentes en edad de cursarla, y el rezago, caracterizado por varios indicadores, tales como el abandono, la reprobación, la repetición de curso y la extra edad, entre otros. De cara a la pobreza y la desigualdad, el Estado mexicano enfrenta el gran reto de continuar ampliando la cobertura para garantizar el acceso equitativo al nivel y la mejora de la calidad, a través de políticas educativas y sociales que logren concretar progresos sustanciales en esta directriz.

Palabras Clave: Rezago educativo, equidad en educación, educación secundaria en México, educación básica.

\section{ABSTRACT:}

This work represents an approach to the situation of Mexican secondary education, which has in recent decades, been experiencing a tension between expanding coverage for all adolescents in age of studying, and the educational gap, characterized by several indicators such as dropout, failure, grade repetition, overage among others. Regarding poverty and inequality, the Mexican government must deal with the challenge of continuing to expand coverage in order to ensure equitable access to the level and improving quality through educational and social policies that can determine substantial advances in this direction.

KEYWORDS: educational gap, educational equity, secondary education in Mexico, basic education.

El artículo que aquí se presenta consiste en un análisis de la situación que experimenta la secundaria mexicana, comprendida como una tensión entre el reto de ampliar la cobertura como derecho universal a la educación de todas las personas, y el rezago que enfrenta a través de varios indicadores como el abandono, la reprobación, la extra edad, entre otros indicadores que se amplifican en algunas de las modalidades instauradas en las zonas marginadas y rurales, donde residen los grupos poblacionales más vulnerables. Esta situación da cuenta de la inequidad que prevalece en el servicio educativo y expresa una de las caras de las desigualdades sociales, económicas y culturales del país, en virtud de que el Estado no ha logrado instaurar programas compensatorios a fin de que estudiantes que provienen de grupos desfavorecidos puedan subsanar las desigualdades de origen a través de la escolarización.

\section{NotAS DE AUTOR}

[2] Doctora en Pedagogía por la Universidad Nacional Autónoma de México. Pertenece al Sistema Nacional de Investigadores. Es investigadora del Instituto de Investigaciones sobre la Universidad y la Educación y profesora en la Facultad de Filosofía y Letras de la UNAM. Es presidenta de la sección mexicana de la Asociación Francófona Internacional de Investigación en Ciencias de la Educación (AFIRSE), cuya matriz se encuentra en Francia. Sus líneas de trabajo son: formación de docentes y formación profesional en la educación; orígenes de la institucionalización de la pedagogía en la universidad de México; educación secundaria; pensamiento crítico en educación y problemática teórica y epistemológica de la investigación en educación; educación básica. Su más reciente publicación es "La investigación en educación: Epistemologías y metodologías". Otras publicaciones: "Quehaceres y saberes educativos del Porfiriato", “Tutoría y mediación I y II” y "Pensamiento crítico en educación" 
Este trabajo parte de la visión de la justicia social del teórico John Rawls, quien utiliza como categoría fundamental de su obra la justicia como equidad, categoría que es retomada para el análisis en el ámbito de la educación secundaria mexicana.

Para la revisión de los indicadores educativos se utilizaron las fuentes oficiales, a partir de las cuales se construyeron algunas tablas que reflejan, por un lado, el crecimiento de la matrícula y, por otro, varios de los rasgos que conforman el llamado rezago educativo, los cuales muestran las brechas entre el alumnado adolescente que logra el egreso exitoso del nivel y el que evidencia problemas en su trayectoria escolar.

El artículo se encuentra estructurado con base en cuatro apartados: se abre este texto con un esbozo de las particularidades de la secundaria mexicana en el marco del sistema educativo nacional; en el segundo apartado se plantea un acercamiento a la noción de equidad desde la teoría de Rawls; en el tercero se exhibe el logro alcanzado por el gobierno en materia de ampliación de la cobertura y, en el cuarto, se muestra la inequidad en cuanto al acceso, la retención y el egreso exitoso de estudiantes, como desafíos que enfrenta el Estado. En el cierre del texto se presentan algunas consideraciones finales.

\section{LA SECUNDARIA MEXICANA}

De acuerdo con la Clasificación Internacional Normalizada de la Educación (CINE) del Instituto de Estadística de la Organización de las Naciones Unidas para la Educación, la Ciencia y la Cultura [ (NE) 0e la Educación [ Educaciecios de los materiales solicitados por el aumento en el tipo de cambio.UNESCO] y la Organización de la Naciones Unidas [ (NE) 0e la Educación [ Educaciecios de los materiales solicitados por el aumento en el tipo de cambio.ONU] (2013), la educación secundaria mexicana se ubica en el nivel CINE 3, correspondiente a la llamada "secundaria baja" y representa el último segmento de la educación básica. En la actualidad, la educación obligatoria en el país comprende 15 años, tres de preescolar, seis de primaria, tres de secundaria y tres de bachillerato o "secundaria alta". Por tanto, la educación secundaria tiene una duración de tres años que se cursan después de haber concluido la primaria, y constituye el requisito para ingresar al bachillerato o educación media superior (secundaria alta).

Las modalidades de la secundaria, a las que pueden optar el estudiantado egresado de la escuela primaria, son fundamentalmente dos, la general y la técnica. La secundaria general pretende ofrecer una formación humanística, científica, artística y tecnológica básica con el objeto de que el estudiantado egresado adquiera los conocimientos y habilidades fundamentales para devenir sujetos productivos y contribuir al desarrollo de la nación. Con el acontecer de los años, se han venido estableciendo algunas modalidades específicas con el objeto de atender la demanda y ampliar la cobertura, particularmente en poblaciones pequeñas, usualmente rurales y marginadas. De ahí que subsisten, entre otras, la telesecundaria, la indígena, para personas que trabajan, la comunitaria y la comunitaria indígena, la de migrantes, y la telesecundaria indígena. La secundaria técnica se caracteriza por ser bivalente, en tanto que, por un lado, brinda la misma formación que la secundaria general y, por otro, capacita al alumnado en algún ámbito tecnológico, de tal suerte que si este no puede continuar estudiando, sí tenga posibilidades de incorporarse prematuramente al trabajo. Las opciones que aquí se ofrecen son las siguientes: industrial, pesquera, forestal, agropecuaria e indígena.

Es importante subrayar que no todas las entidades del país cuentan con todas las modalidades y tipos, sino que cada Estado ha puesto en marcha las que, conforme a las necesidades contextuales de carácter demográfico, geográfico, económico y cultural, se han requerido.

Aun cuando cada una de las modalidades asume ciertas especificidades, de manera genérica, todas comparten ciertos objetivos que posibilitan la construcción de la identidad estudiantil y la formación de la ciudadanía, y se fundan en los siguientes principios (Gobierno Federal y Secretaría de Educación Pública [SEP], 2011): estudiantes como sujetos centrales del proceso de aprendizaje; la planificación como estrategia para potenciar el aprendizaje; la generación de ambientes favorables para el aprendizaje; el trabajo colaborativo; la evaluación como instrumento para promover la mejora; el desarrollo de competencias; la 
inclusión para atender la diversidad; la vinculación entre los diversos actores de la educación: estudiantes, docentes, personal directivo, padres y madres de familia, y la tutoría como componente fundamental para apoyar a quienes más lo requieran.

Un elemento que también es común para todas las modalidades y, de hecho, para toda la educación básica (preescolar, primaria y secundaria), es el relativo al desarrollo de competencias para la vida, las cuales se promueven a través de la organización de experiencias significativas de aprendizaje. Entre las competencias que se pretende impulsar destacan las siguientes (Gobierno Federal y SEP, 2011): a) para el aprendizaje permanente, lo que significa el dominio de habilidades relativas a la lectura, a la escritura y, en general, a la comunicación, no solo en la lengua nacional sino también en alguna otra, pero también el manejo de destrezas digitales; b) para el manejo de la información, competencias que suponen poder buscar, seleccionar, analizar y valorar la información críticamente; c) para el manejo de situaciones y problemas, que corresponden a la movilización de saberes, actitudes y valores destinados a enfrentar la incertidumbre, a reflexionar, a posicionarse, a tomar decisiones y asumirlas, y d) para la convivencia y la vida en sociedad, que implican capacidades para vivir y promover la democracia, reconocer y valorar al otro ser, y actuar a favor de la paz, el respeto, los derechos humanos y la legalidad.

De acuerdo con la organización de cada escuela y, específicamente, con el personal que atiende cada modalidad, se pueden distinguir las secundarias que están a cargo de una planta docente conformada por personal directivo y diverso profesorado que procede de formaciones disciplinares específicas - matemáticas, historia, español, ciencias, educación física, artística, entre otras-, y de diversas instituciones de educación superior, entre las que destacan las escuelas normales superiores, las de educación física (destinadas particularmente a la formación docente del nivel) y las universidades. Así, mientras las secundarias generales y técnicas cuentan con una heterogeneidad de docentes, las telesecundarias son atendidas, usualmente dependiendo del número de grupos-, por un mismo profesor o profesora, quien está a cargo de todas las asignaturas que conforman el currículo del ciclo y quien, muy frecuentemente, se hace cargo también de la dirección de la escuela.

Desde el punto de vista curricular, tanto las escuelas generales como las técnicas comparten el mismo plan de estudios, no obstante, en estas últimas se adicionan asignaturas que pretenden habilitar al estudiantado en el marco de diversas opciones que lo capaciten para una inserción precoz al mercado laboral.

Desde el punto de vista del sostenimiento, la escuela secundaria puede clasificarse en pública y privada, la primera a cargo del Estado mexicano, mientras que la segunda es financiada por particulares.

En suma, la educación secundaria, antiguamente estructurada para la preparación para el bachillerato o secundaria alta, en la actualidad se configura con base en una función bivalente: por una parte, representa una continuidad de la educación primaria, al ofrecer una formación complementaria a esta, de tal manera que, en el país, se encuentra integrada a la educación básica y; por otra parte, desempeña un papel en la preparación para determinados trabajos, al igual que la secundaria alta, específicamente de los sustratos económicos bajos. De esta forma, este nivel educativo experimenta, a la fecha, una gran tensión entre estas dos orientaciones, al ser terminal para muchos grupos y preparatoria para algunos.

\section{UN ACERCAMIENTO CONCEPTUAL A LA INEQUIDAD}

En la actualidad, la equidad es considerada como una de las categorías nucleares en el análisis de los sistemas educativos en el mundo entero: para los ssectores políticos y para quienes toman las decisiones constituye una preocupación prioritaria ante la situación que, a nivel internacional, experimentan la infancia y la juventud no escolarizada; para docentes, una realidad a enfrentar permanentemente en la vida cotidiana del aula, y para los grupos investigativos, una temática emergente imposible de soslayar.

En efecto, esta dimensión ha sido reiterada a través de varios pronunciamientos y acuerdos de la comunidad internacional. Así se registra que desde que nació el movimiento de "Educación para Todos", con la 
Declaración Mundial de Jomtien (1990) y el Marco de Acción de Dakar (2000), los Estados nacionales se comprometieron desde hace ya varias décadas a "suprimir las disparidades en primaria y secundaria..., en particular garantizando a los jóvenes un acceso pleno y equitativo a una educación básica de buena calidad, así como un buen rendimiento" (UNESCO, 2000, p. 8). Sin embargo, este compromiso lamentablemente no se ha concretado en varias regiones del mundo y, particularmente, en Latinoamérica, incluido México, porque como se ha puntualizado más recientemente: "La exclusión e inequidad que afectan a los alumnos... es muy elevada y generalizada en América Latina y el Caribe, tanto en el acceso, como en la progresión y logros de aprendizaje, en los diferentes ciclos escolares" (UNESCO-SEP, 2013, p. 13). Por ello, la UNESCO continúa subrayando, de manera contundente, esta demanda, al exhortar a todos los países y señalar que "el acceso y el aprendizaje equitativos, en particular para las niñas y las mujeres, debe constituir el núcleo de los programas educativos para después de 2015, a fin de aprovechar plenamente el potencial de todas las personas" (UNESCO, 2015, párr. 8).

¿Qué se entiende por equidad? ¿Equidad e igualdad son sinónimos? ¿Cómo se conceptualiza la equidad en educación? ¿Qué relación se establece entre equidad, igualdad y justicia en educación? ¿Cuáles son los problemas que se enfrentan para avanzar en materia de equidad educativa en México?

Las respuestas a estas preguntas no son obvias ni evidentes. La noción de equidad se advierte compleja de manera genérica, por lo que iniciamos por una breve revisión etimológica: el término proviene del latín "aequitas-atis, originariamente igualdad de ánimo", y, a la vez, de "aequus', que significaba igual e igualdad” (Etimologías filosóficas, 2011, párr. 1). También equidad proviene del griego “epiekeia”, que se

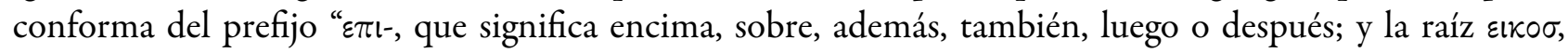
esperable, conveniente, razonable o justo" (Etimologías filosóficas, 2011, párr. 1). Si bien la ley es justa -de acuerdo con Aristóteles- la "epiekeia" lo es más, debido a que esta puede adaptarse a los casos concretos, a las situaciones específicas, es decir, a la singularidad de la situación: "es la expresión de un derecho más profundo y auténtico que el de la ley” (Etimologías filosóficas, 2011, párr. 6).

Según Lalande (1988, pp. 295-296), la equidad es el «sentimiento seguro y espontáneo de lo justo y de lo injusto, especialmente cuando se manifiesta en la apreciación de un caso concreto y particular".

De esta forma, la equidad es comprendida como la interpretación juiciosa, sensata, ponderada, discreta de la ley, lo que significa apartarse, de alguna manera, del sentido literal de esta misma o ir más allá de ella, debido a que esta -la ley universal- no puede prever todos los casos, es decir, la equidad supone atender las particularidades de las personas, conforme al contexto, al tiempo. "Es una forma de justicia que toma más en consideración el espíritu de la ley que la letra, para atenuar los efectos o modificarla, si, como dice Aristóteles, 'se muestra insuficiente debido a su carácter general” (La Dissertation, 2014, párr. 5).

Extrañamente, aunque en todos los países se discute sobre la equidad en educación -en los círculos políticos, académicos y legislativos, sobre todo-, en las enciclopedias y diccionarios especializados no se incluye un tratamiento conceptual sobre la equidad, probablemente por haber devenido una temática más reciente, anclada en la ética y la moral. Son, fundamentalmente, profesionales de economía, filosofía, psicología y juristas quienes han abordado la equidad y la igualdad -tanto desde las teorías clásicas como desde las contemporáneas- en el marco de lo social, del comercio, de la ética, de la administración y del derecho, abonando con sus planteamientos a la búsqueda de la instauración de una justicia social. No obstante, en la actualidad, la equidad se inscribe -cada vez con mayor agudeza- en la esfera de lo educativo, como uno de los ámbitos prioritarios de su adscripción, frecuentemente ligada a la instauración de la justicia social, dimensión, a su vez, articulada a la distribución de la riqueza y a la lucha contra la pobreza.

A fin de continuar reflexionando sobre qué es la equidad, retomo nuevamente a Aristóteles, quien desde la perspectiva de la moral (Moral a Nicómaco), articula la equidad y la justicia, para pasar posteriormente a incursionar de manera breve sobre la equidad en educación.

Aristóteles puntualiza que justicia y equidad no tienen un idéntico significado, pero tampoco son totalmente diferentes. Conceptualiza, por un lado, la equidad como lo bueno, distinto del acto de justicia y, 
por otro, lo equitativo como una noción diferente de lo justo, de tal forma que "el hombre equitativo, que es mejor que el justo en una circunstancia dada, es justo igualmente; y no es porque sea de otro género que lo justo el que sea mejor en el caso dado... y siendo buenos ambos, la única diferencia que hay entre ellos es que lo equitativo es mejor aún" (De Azcárate, 2005, parr. 1). El problema es que al ser lo equitativo justo, no necesariamente es justo de acuerdo con la ley, porque esta -como arriba puntualizamos- es de corte general, y los casos particulares no pueden ser atendidos solamente desde la mirada de la legalidad. En suma, las nociones de equidad y de justicia se encuentran estrechamente vinculadas, aunque, a la vez, y, contradictoriamente, no son lo mismo.

A continuación esbozo el planteamiento de Rawls, quien en su obra clásica La teoría de la justicia (1971) y en su trabajo La justicia como equidad (1999), elabora su propuesta teórica en el marco de una concepción política de la justicia válida y pertinente para una sociedad democrática, cuyas aportaciones bien pueden coadyuvar a reflexionar y comprender la situación de inequidad que subyace en la educación secundaria en el país. Subraya el autor que si bien equidad y justicia no son dos nociones idénticas, la primera es la dimensión prioritaria del concepto de justicia. La justicia es considerada como una de las varias virtudes de las instituciones sociales, es decir, de lo que el autor denomina prácticas, y la igualdad, como un componente de la justicia. En el marco de las prácticas, en donde se despliegan los actores, el concepto de justicia, formulado por el autor, recoge dos principios fundamentales: el principio de igualdad y el principio de diferencia.

a) En relación con el primero subraya que "cada persona debe tener un derecho igual al sistema total más amplio de libertades de base iguales para todos, compatible con un mismo sistema para todos" (Rawls, 1971, p. 341) ${ }^{[3]}$, es decir, todas las personas tienen los mismos derechos a la mayor libertad.

b) El segundo principio refiere a la igualdad de oportunidades y es enunciado así: "las desigualdades económicas y sociales deben ser tales que: a) sirvan para el máximo beneficio de los desfavorecidos y b) estén asignadas a funciones y posiciones abiertas a todos, conforme al principio de la justa igualdad de oportunidades..." (Rawls, 1971, p. 341), lo que significa que las desigualdades no se refieren a las diferencias entre cargos o posiciones, sino a aquellas que son arbitrarias, comprendidas como diferenciaciones en cuanto a beneficios. De ahí que libertad e igualdad integran el concepto de justicia.

Desde esta perspectiva y en el marco de la democracia, Rawls considera que una sociedad justa no es necesariamente igualitaria, pero sí lo es una sociedad equitativa, en la que las ventajas y el provecho alcanzados por algunos grupos, con base en el estatus, concurren para beneficio de los sectores más pobres, es decir, sus beneficios y ventajas son justificados por la utilidad común. De ahí que las desigualdades sociales, étnicas, lingüísticas, culturales, geográficas, físicas, psíquicas de origen pueden ser superadas por acciones compensatorias, de tal forma que se logre corregir la igualdad absoluta, con el fin de alcanzar la justicia, es decir, la equidad. En consecuencia, la noción de equidad solo puede reencontrarse con la de igualdad, cuando esta es conceptuada de cara a la justicia.

Es, sin duda, este razonamiento el que me conduce al análisis del escenario en el que se despliega el servicio educativo en México, particularmente en lo que se refiere a la secundaria básica, la que, como integrante del sistema educativo nacional y de la educación obligatoria, ha participado en la búsqueda de la ampliación de la cobertura en contextos poco favorecidos, donde habita la población más vulnerable. Es ahí, en las zonas distantes, rurales, carentes de servicios básicos y de oportunidades, donde se localizan adolescentes que conforman las minorías socioculturales, provenientes de entornos familiares caracterizados por la pobreza, el trabajo informal, la baja o nula escolarización de los padres y madres, entre otros factores. Pero también es ahí donde el Estado ha pretendido atender la exigencia del derecho a la educación de todas las personas como una responsabilidad básica para el desarrollo de los individuos y de la nación, pero por encima de la accesibilidad, el derecho a la educación implica también el derecho a una educación de buena calidad, adaptada a sus particularidades. 
En México, existen grandes brechas territoriales en lo que al servicio de la secundaria básica se refiere, tanto entre las diferentes entidades que conforman el territorio nacional -entidades que concentran la población más pobre frente a entidades que mantienen mejores niveles de vida- como al interior de cada una de ellas: entre las zonas urbanas y las zonas periféricas, tugurios o zonas rurales, donde residen los grupos minoritarios -indígenas, poblaciones pequeñas, poblaciones en pobreza o pobreza extrema-, lo cual favorece el círculo de la reproducción de las desigualdades económicas, sociales, raciales y educativas.

Esta situación define, en muy buena medida, las brechas en cuanto a la trayectoria escolar de adolescentes y sus logros en aprendizaje, a lo que se adiciona la calidad de la educación que ahí se brinda, lo que alude inevitablemente a la inequidad con la que opera la educación nacional. Lejos de compensar las desigualdades originarias de las poblaciones vulnerables, en el marco de la justicia, el Estado no ha logrado la verdadera inclusión de la población pobre en los ámbitos productivos y sociales, como tampoco en el ámbito educativo, tal como a continuación se esboza en los siguientes apartados, donde se perfilan algunos indicadores que dan cuenta de la tensión existente entre la ampliación de la cobertura y el rezago que prevalece en determinadas modalidades de la secundaria básica, las cuales se encuentran asentadas en las zonas de población vulnerable.

\section{EL LOGRO: EL CRECIMIENTO DEL ACCESO A LA EDUCACIÓN SECUNDARIA}

Con la finalidad de avanzar en la configuración de un sistema educativo equitativo, incluyente y de calidad, la inversión en educación de la población mexicana "es similar al promedio de la OCDE" (Organización para la Cooperación y Desarrollo Económicos [OCDE], 2015) y se ha ido incrementando de manera sistemática: de 1995 a 2000, a una tasa media anual de 6,6\%; de 2001 a 2006, de 3\%, y de 2007 a 2012, de 2,8\% (Instituto Nacional para la Evaluación de la Educación [INEE], 2014). Esta tendencia ha impactado en el continuo crecimiento de la matriculación de niños, niñas y jóvenes, especialmente la de adolescentes de la educación secundaria, dado que ha experimentado un notable incremento a lo largo de las últimas dos décadas.

El inicio de la expansión de este ciclo básico de la educación media, durante la década de los noventa del siglo pasado, es el resultado de la reforma emprendida por la Federación, los gobiernos de los Estados y el Sindicato Nacional de Trabajadores de la Educación [SNTE], instancias todas que firmaron el denominado Acuerdo Nacional para la Modernización de la Educación Básica, en mayo de 1992. A partir de este acuerdo se pretendía atender la demanda insatisfecha abatiendo las desigualdades, y mejorar el servicio educativo con base en tres líneas directrices: la reorganización del sistema educativo, la reformulación de planes y programas y la revaloración social de la función magisterial.

La primera línea obedecía a la necesidad de descentralizar el sistema, otorgando a los Estados recursos y responsabilidades anteriormente atribuidas a la Federación ${ }^{[4]}$. En cuanto a lo que alude a la reformulación de planes y programas, se diseñó un nuevo currículo para la educación básica y se elaboraron materiales educativos diversos tanto para estudiantes como para docentes. Respecto a la revaloración de la función docente, hay que destacar que la escuela normal, que tradicionalmente ha sido la formadora de maestros y maestras de educación básica, a la que se ingresaba después de concluida la escuela secundaria, fue instituida, desde 1984, como institución de educación superior, al ofrecer a partir de entonces licenciatura, a la que se ingresa después de concluido el bachillerato. Sin embargo, la reforma de la formación normalista no se concretó paralelamente a la de educación básica, sino hasta el año 2000.

Por lo que toca a la educación secundaria y, aunque muy tardíamente con respecto a otros países de la región, en la nueva Ley General de Educación (Secretaría de Gobernación, 1993), emitida como efecto del Acuerdo de Modernización, se establece la obligatoriedad de la educación secundaria: "Todos los habitantes del país deben cursar la educación primaria y la secundaria. Es obligación de los mexicanos hacer que sus hijos o pupilos menores de edad cursen la educación primaria y la secundaria" (Secretaría de Gobernación, 1993, parr. 8). Por tanto, la escolarización obligatoria, precedentemente de seis años, se extendía, a partir de entonces, a nueve años, aunque, posteriormente, se promulgaría la obligatoriedad de preescolar ( 3 años), en 
$2002^{[5]}$ y la media superior o bachillerato (3 años), en 2012, extendiendo, de esta forma, la escolarización obligatoria a 15 años para todos los niños, niñas y jóvenes del país.

En las últimas décadas y a partir del acto legislativo que establece la obligatoriedad de la secundaria, el crecimiento de la matrícula ha sido espectacular al pasar de 4190190 estudiantes que se registran en el ciclo escolar 1990-1991 a 6571858 en el ciclo 2013-2014, lo que significa un aumento de 56,83\% en menos de 25 años. De las dos grandes modalidades de la secundaria, la técnica es la que ha registrado un mayor crecimiento con respecto a la secundaria general, a excepción del último ciclo escolar señalado, aunque es esta última la que concentra la matrícula más grande, ya que duplica prácticamente la de estudiantes de la técnica. En la Tabla 1 se presenta la matrícula a través de diferentes cursos escolares.

TABLA 1

Matrícula total de la educación secundaria

\begin{tabular}{|c|c|c|c|c|c|c|c|c|c|c|c|}
\hline Modalidad & $\begin{array}{c}1990- \\
1991\end{array}$ & $\begin{array}{l}1995- \\
1996\end{array}$ & $\begin{array}{l}\% \\
\text { (1) }\end{array}$ & $\begin{array}{l}2000- \\
2001\end{array}$ & $\%$ & $\begin{array}{l}2005- \\
2006\end{array}$ & $\%$ & $\begin{array}{l}2010- \\
2011\end{array}$ & $\%$ & $\begin{array}{c}2013- \\
2014\end{array}$ & $\%$ \\
\hline $\begin{array}{l}\text { Secund. } \\
\text { general }\end{array}$ & 3068190 & 3360600 & 9 & 3842681 & 14 & 4295297 & 11 & 4406029 & 2 & 4745235 & 8 \\
\hline $\begin{array}{l}\text { Secund. } \\
\text { técnica }\end{array}$ & 1122000 & 1326735 & 18 & 1506978 & 13 & 1683959 & 11 & 1731517 & 3 & 1826623 & 5 \\
\hline TOTAL & 4190190 & 4687335 & 12 & 5349659 & 14 & 597256 & 12 & 6137546 & 3 & 6571858 & 7 \\
\hline
\end{tabular}

Nota: Los porcentajes fueron redondeados. Fuente: Secretaría de Educación Pública (SEP).

(2015). Serie histórica y pronósticos de la estadística del sistema educativo nacional. México.

Paralelamente al crecimiento de la matrícula, se ha experimentado un aumento enorme de escuelas generales y técnicas, las cuales, en total, casi se han duplicado durante el periodo 1990-1991 a 2013-2014; de las 19228 existentes en el ciclo inicial, se cuenta ahora con 37 924, lo que representa un aumento de 98\% durante este periodo; sin embargo, son las generales las que han registrado el mayor incremento, el cual alcanza un puntaje de $107 \%$. En la Tabla 2 se puede observar la distribución de la matrícula en las dos grandes modalidades de la educación secundaria.

TABLA 2

Matrícula en escuelas secundarias generales y técnicas

\begin{tabular}{|c|c|c|c|c|c|c|c|c|c|c|c|}
\hline Modalidad & $\begin{array}{l}1990- \\
1991 \\
\end{array}$ & $\begin{array}{c}1995- \\
1996 \\
\end{array}$ & $\%$ & $\begin{array}{l}2000- \\
2001 \\
\end{array}$ & $\%$ & $\begin{array}{l}2005- \\
2006 \\
\end{array}$ & $\%$ & $\begin{array}{l}2010- \\
2011 \\
\end{array}$ & $\%$ & $\begin{array}{l}2013- \\
2014 \\
\end{array}$ & $\%$ \\
\hline $\begin{array}{l}\text { Secund } \\
\text { general }\end{array}$ & 16079 & 19877 & 24 & 24378 & 23 & 27732 & 14 & 31386 & 13 & 33270 & 6 \\
\hline $\begin{array}{l}\text { Secund } \\
\text { técnica }\end{array}$ & 3149 & 3560 & 13 & 3975 & 12 & 4280 & 8 & 4535 & 6 & 4654 & 3 \\
\hline TOTAL & $\begin{array}{c}19 \\
228\end{array}$ & 23437 & 22 & 28353 & 21 & 32012 & 13 & 35921 & 12 & 37924 & 6 \\
\hline
\end{tabular}

Nota: Los porcentajes fueron redondeados. Fuente: Secretaría de Educación Pública (SEP).

(2015). Serie histórica y pronósticos de la estadística del sistema educativo nacional. México.

La opción que ha experimentado la mayor expansión entre las diferentes posibilidades de la secundaria es, sin duda, la telesecundaria, particularmente durante la década de los noventa y el inicio de siglo, tal como se muestra en la Tabla 3. El curso escolar 1990-1991, que contaba con 470093 estudiantes, transitó en 
2013-2014 a 1369 638, es decir, registró un incremento de 191\%. Por otro lado, es en los últimos años cuando, a partir de la creación de la secundaria para migrantes, esta acentuó su participación de 2010-2011 a 2013-2014 con un aumento de 300\%. Lo mismo puede subrayarse respecto a la secundaria comunitaria, la cual, en el mismo lapso de tres años, registró un crecimiento de 38\%. Contrariamente, la secundaria para personas que trabajan ha venido descendiendo la matrícula en los últimos años; mientras en el curso 1990-1991 contaba con 105047 estudiantes, para 2013-2014 solo registra 27 722, lo que representa un decremento importante, de $-74 \%$.

TABLA 3

Matrícula distribuida en las diferentes opciones de secundaria

\begin{tabular}{|c|c|c|c|c|c|c|c|c|c|}
\hline Modalidad & $\begin{array}{l}1990- \\
1991\end{array}$ & $\begin{array}{l}2000- \\
2001\end{array}$ & $\%$ & 2005-2006 & $\%$ & $\begin{array}{l}2010- \\
2011\end{array}$ & $\%$ & $\begin{array}{l}2013- \\
2014\end{array}$ & $\%$ \\
\hline General & 2493050 & 2738970 & 5 & 3019160 & 10 & 3085568 & 2 & 3309503 & 7 \\
\hline $\begin{array}{l}\text { Para } \\
\text { trabaj. }\end{array}$ & 105047 & 50249 & -33 & 41415 & - & 30108 & -27 & 27722 & 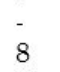 \\
\hline Telesec & 470093 & 1053462 & 52 & 1233862 & 17 & 1262923 & 2 & 1369638 & 8 \\
\hline $\begin{array}{c}\text { Comunitindígena } \\
\text { Comunit }\end{array}$ & $\begin{array}{l}0 \\
0\end{array}$ & $\begin{array}{l}0 \\
0\end{array}$ & $\begin{array}{l}0 \\
0\end{array}$ & $\begin{array}{c}860 \\
0\end{array}$ & - & $\begin{array}{c}0 \\
27240\end{array}$ & - & $\begin{array}{c}0 \\
37620\end{array}$ & $\begin{array}{l}0 \\
38\end{array}$ \\
\hline $\begin{array}{l}\text { Migrant } \\
\text { TOTAL }\end{array}$ & $\begin{array}{c}0 \\
3068190 \\
\end{array}$ & $\begin{array}{c}0 \\
3842681 \\
\end{array}$ & $\begin{array}{c}0 \\
14\end{array}$ & $\begin{array}{c}0 \\
4295297 \\
\end{array}$ & $\overline{12}$ & $\begin{array}{c}190 \\
\mathbf{4} 406029 \\
\end{array}$ & - & $\begin{array}{c}752 \\
\mathbf{4} \mathbf{7 4 5} \mathbf{2 3 5} \\
\end{array}$ & $\begin{array}{l}300 \\
8\end{array}$ \\
\hline
\end{tabular}

Nota: Los porcentajes fueron redondeados. Fuente: Secretaría de Educación Pública (SEP).

(2015). Serie histórica y pronósticos de la estadística del sistema educativo nacional. México.

En cuanto a la secundaria técnica, la mayor concentración de alumnado y el mayor crecimiento de la matrícula durante este periodo se registra en la opción industrial, al pasar de 822 940, en el ciclo 1990-1991 a 1399216 estudiantes, en el de 2013-2014, asentando así un crecimiento porcentual de 70. La distribución de la matrícula con base en las diferentes opciones de la secundaria técnica se puede percibir en la Tabla 4. Después de la industrial, la opción agropecuaria le sigue en cuanto a alumnado matriculado durante estos más de veinte años y, en un tercer lugar, la pesquera. Aunque nunca contó con una gran población escolar, la secundaria indígena no solo no ha crecido, sino que ha disminuido, a excepción del último periodo analizado (2013-2014), en el que tuvo un ascenso relativo al pasar de 68 estudiantes con matrícula, en 2010-2011, a 107 estudiantes, en 2013-2014.

TABLA 4

Matrícula distribuida en las diferentes opciones de la secundaria técnica

\begin{tabular}{|c|c|c|c|c|c|c|c|c|c|}
\hline Modalidad & $\begin{array}{l}1990- \\
1991 \\
\end{array}$ & $\begin{array}{c}2000- \\
2001\end{array}$ & $\%$ & $\begin{array}{l}2005- \\
2006\end{array}$ & $\%$ & $\begin{array}{l}2010- \\
2011\end{array}$ & $\%$ & $\begin{array}{l}2013- \\
2014 \\
\end{array}$ & $\%$ \\
\hline Industri & 822940 & 1123021 & 14 & 1268535 & 13 & 1318958 & 3 & 1399216 & 6 \\
\hline Agropec & 286041 & 366959 & 12 & 397112 & 8 & 393270 & - & 406326 & 3 \\
\hline Pesquer & 8706 & 11352 & 10 & 11455 & 1 & 12137 & 6 & 13165 & 8 \\
\hline Forestal & 3936 & 5514 & 17 & 6757 & 22 & 7084 & 5 & 7809 & 10 \\
\hline Indígena & 377 & 132 & $\begin{array}{c}- \\
49\end{array}$ & 100 & - & 68 & - & 107 & 57 \\
\hline TOTAL & 1122000 & 1506978 & 14 & 1683959 & 12 & 1731517 & 3 & 1826623 & 5 \\
\hline
\end{tabular}

Nota: Los porcentajes fueron redondeados. Fuente: Secretaría de Educación Pública (SEP).

(2015). Serie histórica y pronósticos de la estadística del sistema educativo nacional. México.

También, al igual que el crecimiento de la matrícula en las telesecundarias, el número total de estas escuelas a nivel nacional experimentó un incremento fundamental al pasar de 8423 en 1990-1991 a 18420 en 2013-2014. El porcentaje de crecimiento registrado a lo largo de estas dos décadas asciende a 118\%. A 
su vez, es de destacar que, consecuentemente, la planta docente, en esta modalidad, ha ido aumentando paralelamente al crecimiento de la matrícula y de las escuelas: mientras en 1990-1991 se contaba con un profesorado integrado por 234,293 integrantes, para 2013-2014, este se conforma por 400923.

Desde el punto de vista del sostenimiento de la escuela secundaria, es evidente que, a lo largo de las dos décadas analizadas, el sector público ha aglutinado la mayor matrícula del nivel, comparada con la del sector privado, como se exhibe en la Tabla 5. En el ciclo 1990-1991 registraron 3852297 estudiantes y en el 2013-2014, 6032 618. En tanto, la relativa a la oferta privada transitó de 337893 en 1990-91 a 539240 estudiantes en 2013-2014, aunque destaca el crecimiento correspondiente al ciclo 2000-2001. Durante todo este periodo ambos sectores registraron un aumento porcentual aproximado de 60 .

TABLA 5

Matrícula por sostenimiento de la escuela secundaria

\begin{tabular}{cccccccccc}
\hline Sostenimiento & $\mathbf{1 9 9 0 -}$ & $\mathbf{2 0 0 0 -}$ & $\mathbf{0}$ & $\mathbf{2 0 0 5 -}$ & $\mathbf{2}$ & $\mathbf{2 0 1 0 -}$ & $\mathbf{\%}$ & $\mathbf{2 0 1 3 -}$ & $\mathbf{2 0 1 4}$ \\
& $\mathbf{1 9 9 1}$ & $\mathbf{2 0 0 1}$ & & $\mathbf{2 0 0 6}$ & & $\mathbf{2 0 1 1}$ & $\mathbf{2 0 1 4}$ \\
\hline Pública & 3852297 & 4927611 & 13 & 5531111 & 12 & 5663261 & 2 & 6032618 & 6 \\
Privada & 337893 & 422048 & 28 & 448145 & 6 & 474285 & 6 & 539240 & 14 \\
TOTAL & $\mathbf{4 1 9 0 \mathbf { 1 9 0 }}$ & $\mathbf{5 3 4 9 6 5 9}$ & & $\mathbf{5 9 7 9 2 5 6}$ & & $\mathbf{6 1 3 7 5 4 6}$ & $\mathbf{6 5 7 1 8 5 8}$ & \\
\hline
\end{tabular}

Nota: Los porcentajes fueron redondeados. Fuente: Secretaría de Educación Pública (SEP).

(2015). Serie histórica y pronósticos de la estadística del sistema educativo nacional. México.

Sin embargo, la expansión en este nivel no ha sido la esperada, debido a factores sociales diversos, tales como el origen socioeconómico del alumnado, la ubicación geográfica de la vivienda -rural/urbana-, y el contexto de escolarización de la familia, entre otros. Así vemos que, después de más de 10 años de prescrita la obligatoriedad, para el curso 2005-2006, la cobertura a nivel nacional solo alcanzó 67,9\% de la población en edad de cursar la secundaria, mientras que en el nivel de preescolar (recién expandido como obligatorio a partir de 2004-2005) se registró 51\% ${ }^{[6]}$. Si comparamos estos datos con los relativos al curso 2010-2011, se detectan incrementos importantes más en preescolar que en secundaria. La cobertura en secundaria se acrecentó en tan solo 15 puntos porcentuales, al pasar de 67,9\% a 82,7\%, mientras que la de preescolar aumentó casi 20 puntos porcentuales al transitar de 67,9\% a 82,7\% (INEE, 2006 y 2012).

Para el curso escolar 2013-2014, se registró una cobertura en secundaria de 84,9\%, lo que significa que en estos últimos años el crecimiento fue de apenas dos puntos porcentuales, ya que el grupo poblacional de 12 a 14 años registrado era de 6771326 adolescentes y la matrícula de 5745543 estudiantes. Destaca el dato diferencial relativo al género durante este mismo periodo: mientras la tasa de matriculación en mujeres asciende a $86,5 \%$, la de hombres registra $83,2 \%$, dando una diferencia de tres puntos porcentuales a favor de las primeras (INEE, 2014), probablemente por la anticipada incorporación de aquellos al trabajo.

A nivel nacional y en el marco de las diferentes regiones del país se registra una gran heterogeneidad en materia de cobertura en este nivel educativo para el ciclo 2013-2014; así, se tiene que la Ciudad de México obtiene $100 \%$ de cobertura, y algunas entidades del norte y centro del país registran puntajes elevados tales como 95,1\% Nuevo León; 91,7\% Sonora; 90,7\% Morelos; 89,8\% Zacatecas; 89,4\% Baja California y 89,4\% Coahuila. Inversamente, otros Estados se ubican en el extremo opuesto, particularmente los del sur y sureste, al obtener los más bajos puntajes -Oaxaca 74,4\%; Michoacán 76,4\%; Chiapas 72,2\% y Guerrero 76,6\%-, (INEE, 2014), es decir, las desigualdades en materia educativa prevalecen en tanto que estas últimas entidades se caracterizan por un nivel de desarrollo económico inferior a las del norte y, además, es ahí donde se localiza la mayor presencia de población indígena, población que usualmente habita en comunidades rurales dispersas, de alta o muy alta marginalidad. Es este el principal grupo poblacional que vive debajo de la línea de pobreza, el cual presenta los índices más bajos de desarrollo humano y en donde se aprecian algunos indicadores de la inequidad. 


\section{EL DESAFÍO: LA PROMOCIÓN DE LA IGUALDAD DE ACCESO, RETENCIÓN Y EGRESO}

Si bien, México ha emprendido grandes esfuerzos para promover la equidad en materia educativa al incrementar la inversión en la educación y ampliar la oferta en cuanto a la escolarización de adolescentes en edad de cursar el ciclo básico de secundaria, así como mejorar la calidad del servicio, la escuela secundaria, a diferencia de la primaria, no ha experimentado el crecimiento esperado, es decir, la universalización del ciclo, debido a innumerables factores de corte contextual -socioeconómico y cultural- y, posiblemente, de corte interno -de naturaleza pedagógica (estructura, organización y gestión de la escuela, formación y actualización del profesorado, número de alumnos por grupo, entre otros tópicos)-.

El país está enfrentando, en la actualidad, por un lado, el imponente rezago histórico en materia de escolarización y, por otro, el crecimiento del grupo poblacional de la niñez y adolescencia, ya que, a pesar de la transición demográfica y de los cambios en materia de fecundidad (menor proporción de niños y niñas), tres de cada diez habitantes se ubican entre los 0 y los 14 años de edad; esto significa que, según el censo de 2010, este grupo conforma $30 \%$ de la población total, es decir, treinta y dos y medio millones de niños, niñas y adolescentes que tendrían que tener escolarización en la primaria y la secundaria (Instituto Nacional de Geografía y Estadística [INEGI], 2014). Aun cuando esta cifra, comparada con la de 1990 (treinta y un millón cien mil), no representa un crecimiento excesivo, la gigantesca demanda sí encarna un imponente reto para el gobierno mexicano a fin de poder consolidar la universalización de la secundaria.

A continuación, un acercamiento a algunos de los indicadores que conforman el llamado rezago educativo, y que alertan respecto a la situación de inequidad prevaleciente en la educación secundaria mexicana.

\section{A) El abandono}

De acuerdo con el Fondo de la ONU para la Infancia (UNICEF) y la Comisión Económica para América Latina y el Caribe (CEPAL), aproximadamente $20 \%$ de adolescentes de la región no asiste a la escuela. La mayoría de los niños y las niñas a los 11 años se encuentra matriculada en algún centro educativo, en tanto que a los 17 años, la mitad de adolescentes ya abandonó el sistema educativo; exclusivamente uno de cada tres concluye la secundaria sin rezago, y solamente una persona de cada cinco de la población de ingresos más bajos concluye la secundaria, mientras que la de ingresos más altos logra terminar cuatro de cada cinco (ONU, 2014). En efecto, el abandono constituye uno de los grandes desafíos que enfrenta Latinoamérica y, particularmente, México, cuya presencia se detecta en las poblaciones más vulnerables, en las poblaciones con pocos habitantes y en los grupos indígenas. Por tanto, la situación de la secundaria en el país no es ajena al panorama regional, aun cuando el abandono, también denominado deserción, adopta ciertas particularidades.

Para el Instituto Nacional de Evaluación (INEE), la tasa de deserción es estimada a partir de estudiantes que abandonan la escuela durante el curso de la educación secundaria o en su tránsito hacia el nivel subsiguiente. El mismo organismo establece una distinción entre estudiantado desertor intracurricular y desertor intercurricular. Le denomina deserción intracurricular a la estimada a partir de aquel alumnado que abandonan, durante el transcurso de la educación secundaria, alguno de los tres grados. La deserción intercurricular es la estimada a partir de estudiantado egresado de la educación secundaria que no continúa sus estudios en el nivel educativo subsiguiente ${ }^{[7]}$.

El abandono en la escuela secundaria todavía constituye un gran reto para el Estado Mexicano, porque, si bien, en los últimos años, la tasa estimada ha ido decreciendo, al pasar de 6.8\% en el ciclo 2007-2008, a $5.1 \%$, en el 2012-2013, esto es, 1,7 puntos porcentuales de diferencia, hay todavía un camino que recorrer para lograr la equidad entre la población adolescente. A diferencia de los países asiáticos y africanos, las tasas de deserción total más altas se registran en los hombres, debido, entre otros factores, a su temprana 
incorporación al mercado laboral, pero es de destacar que la brecha de abandono entre hombres y mujeres ha ido descendiendo. La Tabla 6 presenta las diferencias de las tasas de deserción entre hombres y mujeres.

TABLA 6

Tasa de deserción total por género

\begin{tabular}{ccccc}
\hline Año escolar & Total nacional & Hombres & Mujeres & $\begin{array}{c}\text { Diferencias } \\
\text { puntos porcentuales }\end{array}$ \\
\hline $2007-2008$ & 6,8 & 8,3 & 5,3 & 3 \\
$2008-2009$ & 6,4 & 7,6 & 5,2 & 2,4 \\
$2009-2010$ & 6,9 & 7,1 & 4,9 & 2,2 \\
$2010-2011$ & 5,6 & 3,2 & 2,3 & 2 \\
$2011-2012$ & 5,5 & 6,5 & 4,5 & 1,8 \\
$2012-2013$ & 5,1 & 6,0 & 4,2 & 2 \\
\hline
\end{tabular}

Nota: Los porcentajes fueron redondeados. Fuente: Instituto Nacional para la Evaluación de la Educación (INEE)

(2012, 2013 y 2014). Panorama educativo de México. Indicadores del sistema educativo nacional. México.

El abandono intracurricular (durante el transcurso de los tres grados de la educación secundaria), estimado apenas en los últimos años, lejos de haber disminuido entre el curso 2011-2012 al de 2012-2013, se incrementó en 8154 desertores, a diferencia de la deserción total y de la deserción intercurricular, las cuales disminuyeron. En la Tabla 7 se observa la brecha que separa hombres de mujeres, la cual suma 258 estudiantes. En el caso de las adolescentes mexicanas, la situación es muy diferente con respecto a la de los hombres, ya que México, de acuerdo con la OCDE (2015), es el país que registra la mayor tasa de madres adolescentes de entre los países integrantes - una hija o hijo nacido por cada 15 mujeres de 15 a 19 años-, fenómeno posiblemente vinculado con la alta tasa de mortalidad infantil, que representa cuatro veces el promedio de la OCDE: 5,4 infantes mueren por cada 1000 nacimientos (OCDE, 2015), lo que hace plantear como hipótesis: las adolescentes abandonan la secundaria, debido a matrimonios o embarazos precoces y que, además, se dedican posteriormente al cuidado de su progenie. Inversamente, en el tránsito de la secundaria al bachillerato, los desertores son los hombres, duplicando casi a las mujeres.

TABLA 7

Deserción total, intracurricular e intercurricular

\begin{tabular}{|c|c|c|c|c|c|c|c|c|c|}
\hline $\begin{array}{l}\text { Curso } \\
\text { escolar }\end{array}$ & $\begin{array}{c}\text { Deserció } \\
\mathbf{n} \\
\text { Total } \\
\end{array}$ & Hombres & Mujeres & $\begin{array}{c}\text { Deserción } \\
\text { Intra- } \\
\text { curricular }\end{array}$ & Hombres & Mujeres & $\begin{array}{c}\text { Deserción } \\
\text { inter- } \\
\text { curricular }\end{array}$ & Hombres & Mujeres \\
\hline $\begin{array}{l}2011- \\
2012\end{array}$ & 339858 & 204069 & 135789 & 195242 & 116660 & 78582 & 144616 & 87409 & 57207 \\
\hline $\begin{array}{c}2012- \\
2013\end{array}$ & 325692 & 193590 & 132102 & 203396 & 120293 & 82473 & 122296 & 72667 & 49629 \\
\hline Diferencia & -14166 & -10479 & -3687 & +8154 & +3633 & +3891 & -22320 & -14742 & -7578 \\
\hline
\end{tabular}

Instituto Nacional para la Evaluación de la Educación (INEE). (2013 y 2014).

Panorama educativo de México. Indicadores del sistema educativo nacional. México.

En suma, la secundaria mexicana representa un foco rojo para el Estado, si consideramos que las diversas políticas y los distintos programas emprendidos desde el establecimiento de la obligatoriedad no han logrado la equidad educativa en cuanto a la permanencia de estudiantes de este grupo etario. ¿La escuela, como 
responsable del nivel, desempeñará un papel definitorio en la incapacidad de retención y, por tanto, en su dimensión igualadora de oportunidades?

\section{B) La inconclusión del nivel}

El capital básico que se adquiere durante la educación secundaria es un factor esencial para mejorar la calidad de vida de la población, en virtud de que, entre otros tópicos, determina en muy buena medida el acceso al empleo, aun cuando sea anticipadamente y, en consecuencia, favorece el desarrollo económico del país. La carencia de capacidades y habilidades mínimas, así como de conocimientos fundamentales, no solo limita la entrada y la estabilidad en el mercado laboral, sino que también impacta en la reproducción persistente intergeneracional de las desigualdades y de la pobreza. "Al igual que en los países desarrollados, en México, la educación desempeña un papel relevante respecto de las oportunidades laborales, ya que a medida que una persona incrementa su escolaridad, también aumenta la posibilidad de estar empleada” (INEE, 2014, p. 382). Baste con revisar las tasas de la población joven en cuanto a contratación estable para fundamentar esa aseveración. Ciertamente, de acuerdo con los datos de 2013 (INEE, 2014), el sector de 15 a 19 años carente de educación básica - preescolar, primaria y secundaria- solo representó 4,9\% de personas con trabajo estable, mientras que quienes sí contaban con este alcanzó 13,6\%, y las que, además, habían concluido la media alta o bachillerato y la superior, respectivamente ascendió a $53 \%$ y $129 \%$.

Esta situación genérica se duplica si se toman en cuenta las zonas sociodemográficas -urbana, semiurbana y rural-. Por ejemplo, los grupos trabajadores con contratación estable que carecen de educación básica participan, en la zona rural, con 5,5\%; en la zona semiurbana, con 10,1\%; y en la zona urbana con 17,6\%. Los grupos con empleo que sí cuentan con educación básica en la zona rural representan 14,3\%, es decir, la brecha que les separa de los primeros es de 9 puntos porcentuales; quienes laboran en la zona semiurbana duplican el puntaje, al alcanzar $20,5 \%$ de participación, lo que significa una diferencia de 10 puntos porcentuales respecto a los que carecen de la básica; finalmente, los que se localizan en la zona urbana ascienden a 36,2\%, con una diferencia de casi 19 puntos porcentuales. La relación positiva existente entre años de escolaridad y acceso y estabilidad laboral es recurrente, de ahí que la no conclusión de la educación secundaria, sobre todo, en los estratos más desfavorecidos para el caso de México, resulta preocupante.

La no conclusión del nivel es comprendida como la tasa estimada de estudiantes que no concluyeron la educación secundaria en el tiempo normativo. Al respecto, existen diferencias considerables entre el sector público y el privado: mientras que en el curso 2007-2008, la secundaria pública registraba 21,6\% de alumnado sin haber concluido el nivel, la privada exclusivamente $7.3 \%$ como total, marcando con ello una brecha de diferencia de 14.3 puntos porcentuales. Aún más, de ese curso escolar al de 2012-2013, la no conclusión en el sector público descendió a 16,2\%, es decir, 5.4 puntos porcentuales, pero el privado registró un decremento sobresaliente, al contar solo con $1.2 \%$ en este último periodo, lo que significa menos 6.1 puntos porcentuales. En ambos ámbitos, la no conclusión tiene mayor presencia en los hombres que en las mujeres y con mayor diferencia en puntos porcentuales en la esfera pública. En la Tabla 8 se visualiza la diferencia entre escuelas públicas y privadas por género. 
TABLA 8

Tasas de no conclusión en la secundaria pública y privada

\begin{tabular}{|c|c|c|c|c|c|c|}
\hline $\begin{array}{c}\text { Curso } \\
\text { Escolar }\end{array}$ & $\begin{array}{c}\text { Pública } \\
\% \\
\text { Total } \\
\end{array}$ & $\begin{array}{c}\text { Pública } \\
\% \\
\text { Hombres } \\
\end{array}$ & $\begin{array}{c}\text { Pública } \\
\% \\
\text { Mujeres }\end{array}$ & $\begin{array}{c}\text { Privada } \\
\% \\
\text { Total } \\
\end{array}$ & $\begin{array}{c}\text { Privada } \\
\% \\
\text { Hombres } \\
\end{array}$ & $\begin{array}{c}\text { Privada } \\
\% \\
\text { Mujeres } \\
\end{array}$ \\
\hline $2007-2008$ & 21,6 & 26,5 & 16,5 & 7,3 & 8,7 & 5,9 \\
\hline 2008-2009 & 19,7 & 23,2 & 16,0 & 4,8 & 5,5 & 4,1 \\
\hline $2009-2010$ & 18,8 & 22,3 & 15,2 & 4,9 & 6,0 & 4,0 \\
\hline $2010-2011^{8}$ & 17,9 & 21,0 & 14,6 & 2,7 & 4,2 & 1,3 \\
\hline $2012-2013$ & 16,2 & 18,9 & 13,3 & 1,2 & 2,2 & 0,1 \\
\hline
\end{tabular}

Nota: Los porcentajes fueron redondeados. Fuente: Instituto Nacional para la Evaluación de la Educación (INEE). (2012, 2013 y 2014). Panorama educativo de México. Indicadores del sistema educativo nacional. México.

\section{C) El egreso, la reprobación y la repetición}

Comprendida como el alumnado aprobado y aquel que habiendo reprobado acreditaron alguna o varias materias a través de exámenes extraordinarios en los periodos de regularización, la tasa de egreso se ha ido incrementando durante los últimos años, a excepción del curso 2006-2007 en que se registró un leve decremento: de 90,2\% en el curso 2005-2006, a 94,4\% en 2012-2013; el aumento registrado de 4,2 puntos porcentuales que sí es importante, no es suficiente, pues se queda más de $5 \%$ de la matrícula en situación de rezago. Hay que subrayar que las mujeres han llevado la delantera de manera casi constante en cuanto a egreso, aunque los hombres han avanzado en su nivel de participación al pasar de 88,2\% en 2005-2006, a 93,3\%, en 2012-2013, lo que significa un aumento de 5,1 puntos porcentuales; por su parte, las mujeres también acrecentaron el egreso en una proporción más reducida: 3,4 puntos porcentuales del primer curso señalado al de 2012-2013, aunque en este último experimentaron una breve disminución. No obstante, las adolescentes superan en este último periodo en 2.2 puntos porcentuales a los hombres, brecha que ha experimentado un decremento a lo largo de estos años, debido a que en el primero periodo aludido, la diferencia era mayor: 3.9 puntos porcentuales, como se registra en la Tabla 9.

Las diferentes entidades del país reproducen, en materia de egreso, la notable heterogeneidad que les singulariza, ya que, a pesar de que hayan sido los grupos adolescentes más pobres los que han sido beneficiados con la institucionalización de diferentes opciones para el nivel -secundaria comunitaria, telesecundaria, de migrantes, entre otras-, estos no siempre pueden concluirlo por la necesidad de contribuir económicamente a los gastos familiares. 
TABLA 9

Tasa de egreso

\begin{tabular}{|c|c|c|c|c|}
\hline Curso escolar & $\begin{array}{c}\text { Total } \\
\%\end{array}$ & $\begin{array}{c}\text { Incremento } \\
\text { puntos } \\
\text { porcentuales }\end{array}$ & $\begin{array}{c}\text { Hombres } \\
\%\end{array}$ & $\begin{array}{c}\text { Mujeres } \\
\%\end{array}$ \\
\hline $2005-2006$ & 90,2 & - & 88,2 & 92,1 \\
\hline 2006-2007 & 90,1 & $-0,1$ & 88,1 & 92,1 \\
\hline $2007-2008$ & 91,5 & 1,4 & 89,6 & 93,3 \\
\hline 2008-2009 & 91,8 & 0,3 & 90,3 & 93,3 \\
\hline $2010-2011$ & 93,4 & 1,6 & 92,1 & 94,7 \\
\hline 2011-2012 & 93,6 & 0,2 & 82,4 & 94,9 \\
\hline $2012-2013$ & 94,4 & 0,8 & 93,3 & 95,5 \\
\hline
\end{tabular}

Instituto Nacional para la Evaluación de la Educación (INEE). (2012, 2013 y 2014).

Panorama educativo de México. Indicadores del sistema educativo nacional. México.

La temática de la reprobación en la educación secundaria y, en general en la educación básica, es un asunto polémico desde el punto de vista de las políticas educativas. Se pueden detectar prácticas diversas en este rubro. Vaniscotte (1999), en su tipología sobre la estructura de los sistemas educativos europeos, establece algunas diferencias respecto a la reprobación y el fracaso escolar. En la primera, la escuela única de Escandinavia -conformada por Dinamarca, Suecia, Noruega, Islandia y Finlandia-, no existen exámenes, aprobados ni reprobados, ni fracaso escolar; lo que se busca es la realización, el desarrollo y la autonomía del alumnado. En el segundo grupo, la escuela británica -en la que participan Inglaterra, Escocia, Países de Gales e Irlanda-, el estudiantado debe presentar exámenes regularmente, pero no conducen de ninguna manera a la repetición de un grado escolar, porque lo que finalmente interesa es la evaluación del sistema educativo y, también, como en la escuela escandinava, el desarrollo y la autonomía de la niñez. En la tercera estructura denominada “escuela de opciones" - que agrupa a Alemania, Austria, Suiza, Países-Bajos y Bélgica-, el problema de la reprobación no se registra con gran intensidad, debido a que gracias a las opciones técnicoprofesionalizantes, este sistema "modula el nivel de exigencia y limita los riesgos de fracaso" (Vaniscotte, 1999, p. 117). Finalmente, el cuarto tipo de sistema educativo y que el autor intitula "el tronco común de los países latinos y mediterráneos” -aludiendo a Francia, Italia, España, Grecia y Portugal-, concede una gran relevancia a los controles, los exámenes y las evaluaciones, por lo que aquí se registra más habitualmente la reprobación y el fracaso escolar. En cuanto a la educación secundaria, México ha heredado históricamente esta tradición de la reprobación de estudiantes.

Se puede afirmar, en consecuencia, que el tópico de la reprobación escolar no está resuelto a nivel internacional, mientras algunos sectores promulgan sus beneficios, otros apuntan sus limitaciones e inconvenientes, generalizando, por tanto, la aprobación automática en la educación obligatoria. En la región, en varios países existen las prácticas de reprobación por grado escolar o al finalizar el ciclo, al considerar que el alumnado no obtuvo los logros establecidos, e instrumentan programas compensatorios para lograr la aprobación. Sin embargo, las calificaciones aprobatorias o reprobatorias no están sujetas a lineamientos definidos y claros, por lo menos en nuestro país, sino que están determinadas por el profesor o profesora al 
cargo de cada materia, a pesar de la existencia de un currículo genérico nacional para el nivel. De ahí que estudiantes que hubieran reprobado con un profesor, podrían haber quedado aprobados con otro.

En el caso mexicano, se puede observar que, de acuerdo con la información de los últimos tres cursos escolares (INEE, 2013 y 2014), el decremento en repetición ha sido relativo, en tanto que de 2012-2013 a 2013-2014 fue de tan solo 319 estudiantes, y aunque el total de quienes repiten no es gigantesco de acuerdo con los existentes (.95\% en 2011-2012 y .89\% en 2012-2013), los más de 50000 alumnado reprobado se estima como futuras generaciones candidatas al abandono del ciclo. En la Tabla 10 se observa la cantidad de alumnado repetidor y la matrícula existente.

TABLA 10

Matrícula existente y repetición

\begin{tabular}{ccccc}
\hline Curso escolar & Matrícula total & Existencia & Egresados & Repetidores \\
\hline $2010-2011$ & 6137546 & 5938300 & 1792240 & - \\
$2011-2012$ & 6167424 & 5972182 & 1786566 & 56761 \\
$2012-2013$ & 6340232 & 6136836 & 1827830 & 54915 \\
$2013-2014$ & 6571858 & - & - & 54519 \\
\hline
\end{tabular}

Fuente: Instituto Nacional para la Evaluación de la Educación (INEE). (2013 y 2014).

Panorama educativo de México. Indicadores del sistema educativo nacional. México.

Las tasas de reprobación que se regularizan a través de exámenes extraordinarios en los periodos correspondientes, y los no regularizados, también han experimentado una disminución, aunque se cuenta con un mayor número de estudiantado reprobado no regularizado, como se evidencia en la Tabla 11 . De 2005-2006 a 2012-2013, este registró un descenso de 3,5 puntos porcentuales, y el regularizado de 1,9 puntos.

TABLA 11

Reprobación de alumnado regularizado y no regularizado

\begin{tabular}{ccccccccc}
\hline Reprobación & $\mathbf{2 0 0 5 -}$ & $\mathbf{2 0 0 6 -}$ & $\mathbf{2 0 0 7 -}$ & $\mathbf{2 0 0 8 -}$ & $\mathbf{2 0 0 9 -}$ & $\mathbf{2 0 1 0 -}$ & $\mathbf{2 0 1 1 -}$ & $\mathbf{2 0 1 2 -}$ \\
& $\mathbf{2 0 0 6}$ & $\mathbf{2 0 0 7}$ & $\mathbf{2 0 0 8}$ & $\mathbf{2 0 0 9}$ & $\mathbf{2 0 1 0}$ & $\mathbf{2 0 1 1}$ & $\mathbf{2 0 1 2}$ & $\mathbf{2 0 1 3}$ \\
\hline $\begin{array}{c}\text { Sin } \\
\text { regularización }\end{array}$ & $18 \%$ & $16,9 \%$ & $16,4 \%$ & $15,9 \%$ & $16,3 \%$ & $15,9 \%$ & $15,4 \%$ & $14,5 \%$ \\
$\begin{array}{c}\text { Con } \\
\text { regularización }\end{array}$ & $9 \%$ & $8,2 \%$ & $8,4 \%$ & $7,5 \%$ & $7,7 \%$ & $7,4 \%$ & $7,4 \%$ & $7,1 \%$ \\
\hline
\end{tabular}

Secretaría de Educación Pública (SEP). (2013). Principales cifras del sistema educativo nacional 2012-2013 (p. 40). México.

\section{D) La extra edad del alumnado}

En pleno siglo XXI, todavía hay estudiantes que presentan sobre edad en la educación secundaria, debido posiblemente a la reprobación, ya sea en la escuela primaria, ya sea en el ciclo mismo, o bien al ingreso efectuado tardíamente. La población vulnerable que vive en situación de pobreza o de pobreza extrema es la que usualmente es más sensible a esta situación, la cual conduce a que su juventud y niñes tengan menos oportunidades de concluir este nivel educativo y, en consecuencia, a engrosar las cifras del rezago. Si bien es cierto que la inclusión en educación representa una tarea para todos los Estados nacionales, también lo es el propiciar estrategias pertinentes de inclusión, que no de homogeneización, porque los grupos adolescentes que presentan extra edad se encuentran en condiciones diferentes de las de sus pares provenientes de clases medias o altas.

Para enfrentar las demandas de aprendizaje propias de la escuela secundaria, estudiantes provenientes de contextos rurales y pobres requieren, a nivel institucional y áulico, de una atención y disposición por parte del profesorado para acompañarles en su trayecto escolar, es decir, de estrategias compensatorias a fin de que la equidad se consolide en el éxito. La lógica prevaleciente de dirigirse "a la media del grupo", atenta contra 
el derecho a la educación, porque finalmente cada alumna y cada alumno es singular y único, como lo es también cada grupo y cada escuela. En consecuencia, docentes y escuelas tienen la obligación de reconocer las diferencias de intereses, ritmos de aprendizaje, capital cultural, de vida, de estratos económicos, y de garantizar el derecho al servicio educativo, no solo en cuanto al acceso, sino sobre todo en la permanencia y el egreso. De ahí que las políticas educativas, al igual que las institucionales, deben concentrar mayormente su atención en las trayectorias del alumnado más vulnerable (con extra edad y quienes han reprobado), el cual se encuentra en riesgo de repetición de curso y, probablemente, de abandono, entre otros.

Como bien sabemos, a medida que avanza el proceso de escolarización, aumentan las probabilidades de matricularse con sobre edad, y, al respecto, la secundaria, como tercer ciclo de la educación básica, acumula muy frecuentemente el registro tardío en la matriculación. Estudiantes que presentan extra edad "grave", como la califica el INEE, son mucho menos de quienes presentan sobre edad "ligera"; sin embargo, constituyen un grupo también vulnerable que requiere atención. La tasa de alumnado con sobreedad grave ha experimentado un descenso de 1,7 puntos porcentuales de 2009-2010 a 2013-2014, aunque todavía en este último curso la cifra de estudiantes es alta. La extra edad grave, en el caso mexicano, no exhibe diferencias importantes en cuanto al grado que se cursa en la secundaria; más bien se mantiene una cierta reducción del curso 2009-2010 a 2013-2014 en los tres grados. En las Tablas 12 y 13 se presentan las tasas de alumnado con extra edad grave.

TABLA 12

Alumnado con sobre edad grave

\begin{tabular}{cccc}
\hline Curso escolar & Matrícula total & Alumnos con sobre edad & $\%$ \\
\hline $2009-2010$ & 6127902 & 350943 & 5.7 \\
$2010-2011$ & 6137546 & 329094 & 5.4 \\
$2011-2012$ & 6167424 & 305796 & 4.9 \\
$2012-2013$ & 6340232 & 281408 & 4.4 \\
$2013-2014$ & 6571858 & 262190 & 4.0 \\
\hline
\end{tabular}

Instituto Nacional para la Evaluación de la Educación (INEE). (2012, 2013, 2014 y 2015).

Panorama educativo de México. Indicadores del sistema educativo nacional. México.

TABLA 13

Alumnado por grado con extra edad grave

\begin{tabular}{|c|c|c|c|c|c|c|c|c|}
\hline \multirow{2}{*}{ AÑo } & \multicolumn{4}{|c|}{ Alumnos con sobre edad } & \multicolumn{4}{|c|}{ Porcentajes } \\
\hline & TOTAL & $1^{\circ}$ & $2^{\circ}$ & $3^{\circ}$ & TOTAL & $1^{\circ}$ & $2^{\circ}$ & $3^{\circ}$ \\
\hline $2010-2011$ & 329094 & 121538 & 111749 & 95807 & 5,4 & 5,6 & 5,5 & 5,0 \\
\hline 2011-2012 & 305796 & 111031 & 104170 & 90595 & 5,0 & 5,1 & 5,0 & 4,7 \\
\hline 2012-2013 & 281408 & 100942 & 95345 & 85121 & 4,4 & 4,4 & 4,6 & 4,4 \\
\hline 2013-2014 & 262190 & 96957 & 88001 & 77232 & - & - & - & - \\
\hline
\end{tabular}

Instituto Nacional para la Evaluación de la Educación (INEE). (2012, 2013, 2014 y 2015).

Panorama educativo de México. Indicadores del sistema educativo nacional. México.

\section{REFLEXIONES FINALES}

Aun cuando México, al igual que otros países de la región, ha alcanzado logros muy favorables en materia de cobertura en la educación secundaria, prevalecen grandes retos para favorecer, particularmente, a las minorías, la igualdad de oportunidades, y contribuir, en el marco de la democracia, a avanzar hacia la configuración de una sociedad justa y equitativa que garantice a toda la niñez y a la población adolescente una formación ciudadana portadora de valores y de responsabilidad social. 
Uno de los rubros pendientes para el país alude a la proporción de estudiantes por docente en secundaria, la cual, según reporta la OCDE, es la más elevada entre los países miembros. "En 2013 había 32 estudiantes por personal docente en las escuelas [públicas] mexicanas de educación secundaria, esto es, el coeficiente más alto entre los países de la OCDE y países asociados, y más del doble que el promedio de la OCDE” (OCDE, 2015 , p. 4). Por otra parte, la calidad del servicio que se ofrece en algunas de las modalidades asentadas en las poblaciones distantes y rurales, donde operan las telesecundarias, las comunitarias, las de migrantes, no es la deseable, dado que usualmente ahí un solo profesor o profesora está a cargo de todas las asignaturas curriculares, situación que da cuenta de la inequidad que se experimenta en las poblaciones más vulnerables. También, el gasto salarial del profesorado en secundaria representa un elemento sustantivo del gasto total por estudiante, respecto del cual, también México se encuentra rezagado, en tanto que este fue el menor de todos los países de la Organización, al destinar solamente 1100 dólares, cuando el promedio de la OCDE en el nivel es de 3200 dólares. En efecto, el personal docente de educación secundaria es retribuido económicamente por hora/clase impartida, lo que implica que, para poder contar con un ingreso medio, tiene que trabajar 40 horas, entre las cuales no se incluyen las destinadas a preparación de los cursos, a reuniones colegiadas, a la revisión y corrección de trabajos, a la atención y acompañamiento personalizado de estudiantes, a la formación continua, tal como se experimenta en otros países.

La ampliación de la cobertura temprana y la disminución de la reprobación y el abandono solo podrán darse en la medida en que se inviertan más recursos en este ciclo educativo, a fin de mejorar la calidad del servicio, y se definan y operen políticas educativas tendientes a prevenir tempranamente el rezago, ubicando y atendiendo de manera oportuna a estudiantes que se encuentran en riesgo, con personal docente capacitado y comprometido. Será esta una vía estratégica para contribuir a la reducción de las desigualdades en una sociedad que se promete inclusiva.

Las políticas educativas deben invertir la lógica con la que han operado durante las últimas décadas: los mejores maestros y maestras deberían ser asignarse a las escuelas cuyo rezago es mayor; los mayores recursos financieros deberían estar destinados a los centros escolares más vulnerables. Solo así podríamos hablar de justicia social en la educación.

\section{REFERENCIAS}

De Azcárate, P. (2005). Moral a Nicómaco. De la equidad. Recuperado de http://www.filosofia.org/cla/ari/ azc01146.htm

Etimologías filosóficas. (2011). Equidad. Recuperado de http://etimologiaspalomar.blogspot.mx/2011/10/ equidad.html

Gobierno Federal y Secretaría de Educación Pública (SEP). (2011). Plan de Estudios. México. Recuperado de http:// basica.sep.gob.mx/dgdc/sitio/pdf/PlanEdu2011.pdf

Gobierno Federal y Sindicato Nacional de Trabajadores de la Educación (SNTE). (1992). Acuerdo Nacional para la Modernización de la Educación. Recuperado de http://www.sep.gob.mx/work/models/sep1/Resource/ b490561c-5c33-4254-ad1c-aad33765928a/07104.pdf

Instituto Nacional de Estadística y Geografía (INEGI). (2014). Perfil sociodemográfico de niños. México. Recuperado de http://www.inegi.org.mx/prod_serv/contenidos/espanol/bvinegi/productos/censos/ poblacion/2010/perfil_socio/ninos/702825056629.pdf

Instituto Nacional para la Evaluación de la Educación (INEE). (2006). Panorama educativo de México. Indicadores del sistema educativo nacional. México. Recuperado de http://www.inee.edu.mx/images/stories/Publicaciones/ Panorama_educativo/2006/Partes/panorama200605.pdf

Instituto Nacional para la Evaluación de la Educación (INEE). (2012). Panorama educativo de México. Indicadores del sistema educativo nacional 2011. México. Recuperado de http://www.guiaeduca.com.mx/documentos/ educacion/INEE-Panorama-Educativo-de-Mexico-2011.pdf 
Instituto Nacional para la Evaluación de la Educación (INEE). (2013). Panorama educativo de México. Indicadores del sistema educativo nacional 2012. México. Recuperado de http://www.guiaeduca.com.mx/documentos/ educacion/INEE-Panorama-Educativo-de-Mexico-2011.pdf

Instituto Nacional para la Evaluación de la Educación (INEE). (2014). Panorama educativo de México. Indicadores del sistema educativo nacional 2013. México. Recuperado de http://publicaciones.inee.edu.mx/buscadorPub/ P1/B/112/P1B112.pdf

Instituto Nacional para la Evaluación de la Educación (INEE). (2015). Panorama educativo de México. Indicadores del sistema educativo nacional 2014. México. Recuperado de http://publicaciones.inee.edu.mx/buscadorPub/ P1/B/113/P1B113.pdf

La Dissertation. (2014). L'égalité Critère De La Justice. Recuperado de https://www.ladissertation.com/Philosophie/ La-Libert\%C3\%A9/L'\%C3\%A9galit\%C3\%A9-Crit\%C3\%A8re-De-La-Justice-163146.html

Lalande A. (1998). "Équité" Vocabulaire Technique Critique de la Philosophie. 16, 295-296. Paris: Presse Universitaire de France.

Organización de las Naciones Unidas (ONU). (2014). La CEPAL y la UNICEF piden políticas públicas que apoyen la educación secundaria. Centro de Noticias ONU. Recuperado de http://www.un.org/spanish/News/story.asp? NewsID=29087\#.VvNbeOncc0Q.

Organización de las Naciones Unidas (ONU) y Organización de las Naciones Unidas para la Educación, la Ciencia y la Cultura (UNESCO) e Instituto de Estadística. (2013). Clasificación Internacional Normalizada de Educación CINE 2011. Montréal: Autor.

Organización de las Naciones Unidas para la Educación, la Ciencia y la Cultura (UNESCO). (2000). Marco de acción de Dakar. Educación para todos: cumplir nuestros compromisos comunes. Francia: UNESCO. Recuperado de http://unesdoc.unesco.org/images/0012/001211/121147s.pdf

Organización de las Naciones Unidas para la Educación, la Ciencia y la Cultura (UNESCO). (2015). Foro mundial sobre la educación 2015. Educación de calidad, equitativa e inclusiva, así como un aprendizaje durante toda la vida para todos en 2030. Transformar vidas mediante la educación. Corea. Recuperado de http://es.unesco.org/ world-education-forum-2015/5-key-themes/equidad

Organización de las Naciones Unidas para la Educación, la Ciencia y la Cultura (UNESCO) y Secretaría de Educación Pública (SEP). (2013). Situación educativa de América Latina y el Caribe. Hacia una educación para todos, Santiago de Chile. Recuperado de http://www.unesco.org/new/fileadmin/MULTIMEDIA/FIELD/Santiago/ pdf/situacion-educativa-mexico-2013.pdf

Organización para la Cooperación y Desarrollo Económicos (OCDE). (2015). México. Nota país. Panorama de la Educación 2013: Indicadores de la OCDE. Recuperado de https://www.oecd.org/mexico/Education-at-aglance-2015-Mexico-in-Spanish.pdf

Rawls, J. (1971). Théorie de la justice. Paris: Seuil (Points)

Rawls, J. (1999). Justicia como equidad. España: Tecnos. Recuperado de https://dialnet.unirioja.es/descarga/ articulo/1069286.pdf

Secretaría de Educación Pública (SEP). (2013). Principales cifras del sistema educativo nacional 2012-2013. México. Recuperado de http://fs.planeacion.sep.gob.mx/estadistica_e_indicadores/principales_cifras/ principales_cifras_2012_2013_bolsillo.pdf

Secretaría de Educación Pública (SEP). (2015). Serie histórica y pronósticos de la estadística del sistema educativo nacional. México. Recuperado de http://www.snie.sep.gob.mx/estadisticas_educativas.html.

Secretaría de Gobernación. (1993). Ley General de Educación. Recuperado de http://dof.gob.mx/nota_detalle.php? codigo $=4759065 \&$ fecha $=13 / 07 / 1993$.

Vaniscotte, F. (1999). L'enseignant des écoles de l'Europe. Perspectives de Recherche, Perspectives documentaires en e\#ducation, 46-47, 115-124. Recuperado de http://ife.ens-lyon.fr/publications/edition-electronique/ perspectives-documentaires/RP046-14.pdf 


\section{Notas}

[1] Este trabajo forma parte del Proyecto de Investigación intitulado "La educación secundaria: Retos y perspectivas al inicio de siglo", que es financiado por la Dirección General de Personal Académico de la Universidad Nacional Autónoma de México (UNAM), en el marco del Programa de Apoyo a Proyectos de Investigación e Innovación Tecnológica.

[3] Traducción personal del francés.

[4] Tal como se estableció: "A fin de corregir el centralismo y burocratismo del sistema educativo..., el Gobierno Federal y los gobiernos de las entidades... celebran convenios para concretar responsabilidades en la conducción y operación del sistema de educación básica y de educación normal. ...corresponderá a los gobiernos estatales encargarse de la dirección de los establecimientos educativos con los que la Secretaría de Educación Pública ha venido prestando, en cada estado y bajo todas sus modalidades y tipos, los servicios de educacio\#n preescolar, primaria, secundaria y para la formacio\#n de maestros... En consecuencia, el Ejecutivo Federal traspasa y el respectivo gobierno estatal recibe los establecimientos escolares con todos los elementos de cara\#cter te\#cnico y administrativo, derechos y obligaciones, bienes muebles e inmuebles..." (Gobierno Federal y SNTE, 1992).

[5] Con base en la modificación del artículo $3^{\circ}$. Constitucional en 2002, aunque se prescribió su incorporación gradual a la educación básica para los ciclos 2004-2005 y 2008-2009.

[6] No aludimos a primaria porque este nivel educativo alcanza prácticamente $100 \%$ de cobertura.

[7] El Instituto Nacional de Evaluación para la Educación obtiene las tasas de deserción considerando la matrícula inicial del ciclo escolar, el alumnado egresado en el ciclo y el alumnado de nuevo ingreso. (niv) - t+1 ANI(niv) x100 t M1 acivo subsiguiente. alumnos que durante la educaciegor(niv) - $t+1$ ANI(niv) x100 t M1 acivo subsiguiente. alumnos que durante la educaciegor

\section{BY-NC-ND}

